\title{
Severe in utero herpes simplex virus infection in a fetus of an asymptomatic mother
}

\author{
Lindsey A McAlarnen, Sachin Amin and Jean Ricci Goodman* \\ Divisions of Maternal-Fetal Medicine and Neonatology, Departments of Obstetrics and Gynecology and Pediatrics, Loyola University Medical Center, Maywood, \\ Illinois, USA
}

\begin{abstract}
Herpes Simplex Virus (HSV) is rare in neonates but is associated with significant morbidity and mortality. A lack of universal screening coupled with a difficult clinical diagnosis and the need for prompt initiation of antiviral medications can lead to devastating consequences for untreated HSV infections in neonates. Approximately twenty cases of in utero transmission of neonatal HSV have been reported in asymptomatic mothers in the literature. Here we describe such a case of severe in utero transmission of HSV to a fetus born to an asymptomatic mother without symptoms or prior exposure to HSV personally or in her partner. Our patient's atypical, non-vesicular skin lesions and presence of the historical triad of neurological, cutaneous, and eye abnormalities highlight the unique nature of this case.
\end{abstract}

Abbreviations: HSV: Herpes Simplex Virus; ACOG: American College of Obstetrics and Gynecology.

\section{Introduction}

In utero congenital Herpes Simplex Virus (HSV) transmission is rare, accounting for only $5 \%$ of all neonatal herpes infections, with an estimated 1 in 300,000 newborns in the United States affected $[1,2]$. The classic description of in utero HSV is a distinct triad of cutaneous, neurologic, and eye abnormalities [3,4]. However, only one-third of those affected with in utero HSV actually have this classic triad $[5,6]$. Because presentation may vary from disseminated disease to a more subtle presentation, particularly with regard to cutaneous manifestations, delayed diagnosis of neonatal HSV is not uncommon [6]. Prompt institution of antiviral therapy to neonates with HSV appears to improve survival and decrease morbidity, but given the rarity of congenital HSV and the varied presentation, a high index of suspicion is required to make a timely diagnosis [6]. We describe a case of an in utero acquired severe neonatal HSV infection, with delivery by cesarean delivery for other indication, to an asymptomatic mother without history or known exposure to HSV. The neonate's mother provided written consent for reporting of this case and photographs presented.

\section{Case}

A 25-year-old Caucasian G3P2002 was received in transfer to our tertiary level center due to preterm premature rupture of membranes at 35 weeks 2 days gestation, with history of an abnormal outside fetal ultrasound two days prior to transfer. The patient initiated prenatal care at $6 \mathrm{wk} 6 \mathrm{~d}$ gestation, with dates confirmed by first trimester ultrasound. A 20-week routine anatomy scan did not identify structural abnormalities, and growth was concordant with dates. At $32 \mathrm{wk} 5 \mathrm{~d}$ gestation, the patient was diagnosed with gestational diabetes by standard criteria, which was diet controlled until delivery. At $35 \mathrm{wk} 0 \mathrm{~d}$, a growth ultrasound was performed due to gestational diabetes, which revealed a head circumference $<3^{\text {rd }} \%$, an enlarged cisterna magna, small cerebellum and overall estimated fetal weight at the $76^{\text {th }} \%$. The patient reported she was in a monogamous relationship and had no history of sexually transmitted infections, nor did her partner. Breech presentation was determined on admission, the fetal heart tracing was Category 1, and a cesarean section was performed with meconium identified on uterine cavity entry. A live born infant was delivered, with positive pressure ventilation required at delivery. Apgar score was 5 and 7 at 1 and 5 minutes respectively, and arterial cord $\mathrm{pH}$ was 7.21 with a base excess of -8 . Postoperative course was uneventful for the mother, being discharged to home on the fourth post-operative day. Bacterial culture of the patient's placenta was negative. Maternal HSV serologies returned with a positive HSV 2 IGG and negative HSV 2 IGM.

Physical examination of the infant on delivery was significant for microcephaly (head circumference of $29 \mathrm{~cm}<3^{\text {rd }}$ percentile). The baby weighed 2450 grams ( $50^{\text {th }}$ percentile) and had a length of $48 \mathrm{~cm}\left(75^{\text {th }}\right.$ percentile) at birth. Several large scaly plaques with well-demarcated irregular borders were noted on the neck, shoulders, upper chest and back, left proximal arm, scalp and face (Figure 1). Plaques on the forehead were mildly atrophic; those on the trunk had a fine scale, and on the cheek the plaques were eroded. Initial workup consisted of complete blood count, blood culture, surface cultures for HSV, and brain MRI. Herpes surface cultures from the skin lesions, were positive for HSV type 2. A cerebrospinal fluid (CSF) examination was negative; HSV polymerase chain reaction (PCR) study sent from the CSF was also negative. Magnetic resonance imaging (MRI) of the brain was severely abnormal, with cystic encephalomalacia in the bilateral

*Correspondence to: Jean Ricci Goodman, MD, MBA, Professor, Division of Maternal Fetal Medicine, Department of Obstetrics and Gynecology, Loyola University Chicago Stritch School of Medicine, 2160 S. 1st Avenue, Maywood, IL 60153, USA, Tel: 708-216-5459; Fax: 708-216-5057; E-mail: jrgoodman@lumc.edu

Key words: herpes simplex virus, congenital infection, in-utero transmission

Received: November 07, 2018; Accepted: November 16, 2018; Published: November 19, 2018 
frontal and temporal lobes, and in the posterior fossa affecting almost the entire cerebellum (Figure 2). The scattered distribution and cysticappearing encephalomalacia throughout many areas of the brain was consistent with a prior infectious process. There was no definite abnormal enhancement on MRI to suggest active infection. Blood and CSF cultures were negative for bacteria. Ophthalmologic examination revealed retinitis involving the macular area bilaterally. The baby was treated with intravenous acyclovir for a total of 21 days, initiated given high index of suspicion at delivery and continuing in view of confirmed HSV 2 infection with neurologic involvement. The baby was initiated on enteral feeds, but ultimately underwent G-tube placement due to poor oral intake. An echocardiogram revealed a patent ductus arteriosus and partial aortic obstruction suspected due to extension of ductal tissue in to the aorta. Therefore, surgical ligation of the patent ductus arteriosus was performed. The baby was discharged home on oral suppressive acyclovir therapy.

\section{Discussion}

The rate of neonatal HSV infection varies widely dependent on the population studied, as well as given it is not a reportable disease.

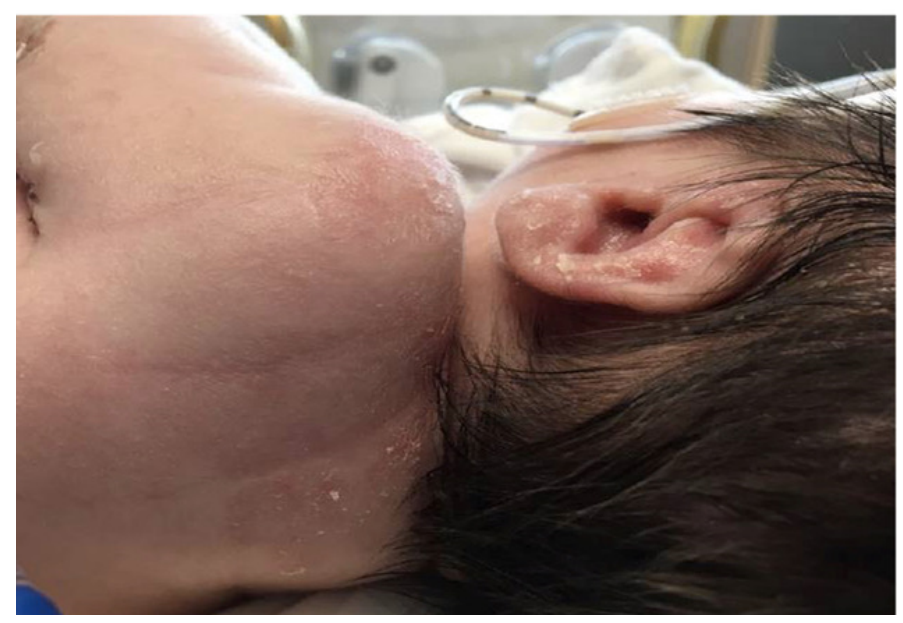

Figure 1. Neonatal day 2, plaques with fine crust and scale on back, shoulder, left ear

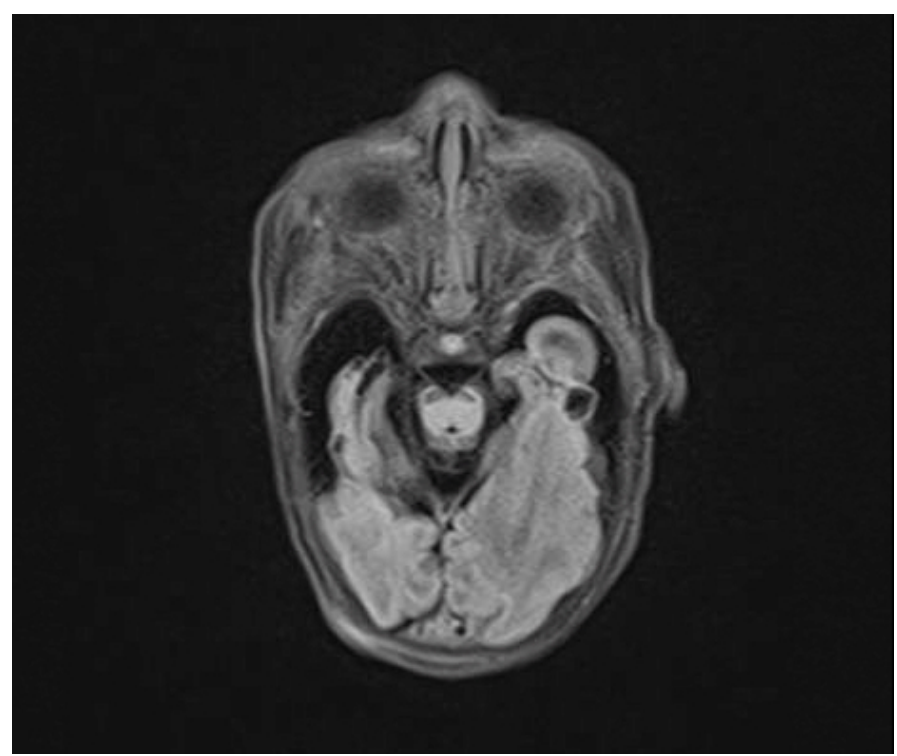

Figure 2. MRI axial T1 post contrast. Neonatal day 2, cystic-appearing encephalomalacia, no abnormal enhancement
Globally, the rate of neonatal herpes has been estimated as ten cases per 100,000 , which is higher than that of syphilis, toxoplasmosis or rubella [2]. Between 1200-1500 cases of neonatal HSV occur in the United States annually, with HSV-2 accounting for approximately twothirds of these cases $[1,3]$. The seroprevalence rate of HSV-2 in the United States general adult population with no exposure or symptom history is estimated to be $16 \%$ [1]. In a prospective cohort study of over 50,000 pregnant women who had their HSV serologic status tested, an HSV seronegative woman had the highest risk of neonatal herpes of 54/100,000 live births, presumed due to their infants lack of type-specific trans placental antibodies [5]. Of the 64 cases of in utero neonatal herpes reported to date, one-third of those mothers lacked a personal or partner history, as in our patient's case [6].

While uncommon, HSV is an aggressive teratogen with destructive potential in the developing brain, as exemplified in our case. Disseminated disease is seen in $25 \%$ of neonatal HSV cases at delivery, but more subtle presentations are common regardless of serotype $[3,6]$. Our case exemplifies that cutaneous manifestations for HSV are not always vesicular, and that one must have a high index of suspicion of the varied cutaneous manifestations to make the diagnosis. Thirty percent of infants with disseminated HSV disease, and $4 \%$ with isolated CNS infection will go on to die of their disease [1,2]. Occurring in a completely asymptomatic mother without history of herpes nor known partner risk, this case highlights the potential veracity of maternal acquisition of HSV in the antepartum period.

\section{References}

1. Feltner C, Grodensky C, Ebel C, Middleton JC, Harris RP, et al. (2016) Serological screening for genital herpes: An evidence review for the U.S. Preventive Services Task Force. Rockville (MD), Agency for Healthcare Research and Quality (US). Available from: http://www.ncbi.nlm.nih.gov/books/NBK409117/

2. Looker KJ, Margaret AS, May MT, Turner KME, Vickerman P, et al. (2017) Firs estimates of the global and regional incidence of neonatal herpes infection. Lancet Glob Health 5: e300-e309. [Crossref]

3. ACOG clinical management guideline for Obstetrician-Gynecologists (2007) Management of herpes in pregnancy. Obstet Gynecol 109: 1489-1498.

4. James SH, Sheffield JS, Kimberlin DW (2014) Mother-to-child transmission of herpes simples virus. J Pediatr Infect Dis Suppl: S19-23. [Crossref]

5. Brown ZA, Wald A, Morrow RA, Selke S, Zeh J, et al. (2003) Effect of serologic status and cesarean delivery on transmission rates of herpes simplex virus from mother to infant. JAMA 289: 203-209. [Crossref]

6. Marquez L, Levy ML, Munoz FM, Palazzi DL (2011) A report of three cases and review of intrauterine herpes simplex virus infection. J Pediatr Infect Dis 30: 153-157. [Crossref]

Copyright: (C2018 McAlarnen LA. This is an open-access article distributed under the terms of the Creative Commons Attribution License, which permits unrestricted use, distribution, and reproduction in any medium, provided the original author and source are credited. 